\title{
First discovery of iron line emission generated by low-energy cosmic rays
}

\author{
K. K. Nobukawa ${ }^{* a}$, M. Nobukawa ${ }^{b}$, S. Yamauchi ${ }^{a}$, H. Uchiyama ${ }^{c}$, K. Koyama $^{d}$ \\ ${ }^{a}$ Department of Physics, Nara Women's University, Kitauoyanishimachi, Nara, 630-8506, Japan \\ E-mail: kumikodcc.nara-wu.ac.ip \\ ${ }^{b}$ Faculty of Education, Nara University of Education, Takabatake-cho, Nara, 630-8528, Japan \\ ${ }^{c}$ Faculty of Education, Shizuoka University, 836 Ohya, Suruga-ku, Shizuoka, 422-8529, Japan \\ ${ }^{d}$ Department of Physics, Graduate School of Science, Kyoto University, \\ Kitashirakawa-oiwake-cho, Sakyo-ku, Kyoto, 606-8502, Japan
}

Cosmic rays (CRs) in our galaxy are thought to be generated via diffusive shock acceleration. Gamma-ray observations have revealed that protons and/or electrons are accelerated to energies up to $100 \mathrm{TeV}$ in supernova remnants (SNRs), and hence SNRs are the most plausible source of CR production. Whereas low-energy cosmic rays (LECRs) below the MeV band have important information on the initial acceleration mechanism, there has been very little information on LECRs due to lack of an effective probe. By interacting with neutral iron atoms in the interstellar medium, LECRs produce fluorescent X-ray line emission at $6.40 \mathrm{keV}$. The $6.40 \mathrm{keV}$ line observation can be a probe to investigate LECRs. The Suzaku data analysis discovered the $6.40 \mathrm{keV}$ line emission from several SNRs interacting with molecular clouds (W28, Kes 67, Kes 69, Kes 78, Kes 79, W44 and 3C391). The $6.40 \mathrm{keV}$ line emission would be produced by $\mathrm{MeV}$ protons with the energy density of $>10-100 \mathrm{eV} \mathrm{cm}^{-3}$. Furthermore, we discovered the $6.40 \mathrm{keV}$ line emission from a giant molecular cloud located near the Galactic center. The energy density of $\mathrm{MeV}$ protons is estimated to be $\sim 80 \mathrm{eV} \mathrm{cm}^{-3}$. The diffusion length of $\mathrm{MeV}$ protons is very short, and thus the $\mathrm{MeV}$ protons should be produced in situ. Surprisingly, there is no SNR in the vicinity. The LECRs would possibly be generated by stochastic acceleration via Alfvén turbulence.

35th International Cosmic Ray Conference

10-20 July, 2017

Bexco, Busan, Korea

*Speaker. 


\section{Introduction}

Galactic cosmic rays (CRs) have been thought to be accelerated in shocks of supernova remnants (SNRs). X-ray observations by ASCA revealed that electrons are accelerated to energies of $\sim 100 \mathrm{TeV}$ [四]. Observations of gamma-ray emission via $\pi^{0}$ decay have offered evidence that protons are accelerated in SNRs [వ]. On the other hand, there has been little information of low-energy (below the MeV band) cosmic rays (LECRs). This is because LECRs coming from our galaxy are hardly observed inside the heliosphere due to the solar modulation. Only exception is VoyagerI, which has escaped the heliosphere [B]]. Furthermore, there is no effective probe to indirectly measure LECRs so far.

Along the Galactic plane, there is diffuse X-ray emission which cannot be resolved into discrete sources (the Galactic ridge X-ray emission; GRXE) [四]. The GRXE is accompanied with K-shell lines from neutral iron (at $6.40 \mathrm{keV}$ ), helium-like iron (at $6.68 \mathrm{keV}$ ), and hydrogen-like iron (at $6.97 \mathrm{keV}$ ) [可]. We measured the distributions of these iron $\mathrm{K}$-shell lines and the averaged GRXE spectrum, and found that a significant fraction of the neutral iron emission (the $6.40 \mathrm{keV}$ line) of the GRXE is likely to originate from the interaction between LECRs and molecular clouds (MCs) [ [, 0$]$. We then found that the $6.40 \mathrm{keV}$ line can be a prove to investigate the LECRs.

\section{Method}

When LECRs are bombarded with the interstellar medium, in particular, MCs, the neutral iron line at $6.40 \mathrm{keV}$ is produced via inner-shell ionization of neutral iron, and at the same time, the X-ray continuum is produced via inverse bremsstrahlung (for protons) or bremsstrahlung (for electrons). The cross section of iron K-shell ionization peaks at $\sim 10 \mathrm{MeV}$ and $\sim 10 \mathrm{keV}$ for protons and electrons, respectively [ [ $]$ ]. The cross section for electrons has a sharp cut-off at $7.1 \mathrm{keV}$, while that for protons varies smoothly [ [8]. This difference of cross section results in the difference of the equivalent width (EW) of the $6.40 \mathrm{keV}$ line between protons and electrons. The EW of the $6.40 \mathrm{keV}$ line as a function of particle index is calculated by [ $[8]$ and shown in figure $\mathrm{W}$. Here we adopt the iron abundance of 1-1.5 solar. The $6.40 \mathrm{keV}$ line produced by electrons has the EW below $\sim 600 \mathrm{eV}$, while for protons the $\mathrm{EW}$ can be above $1 \mathrm{keV}$. Thus, we can distinguish the irradiating particles by the EW.

The $6.40 \mathrm{keV}$ line can be produced also by X-rays with the energy higher than $7.1 \mathrm{keV}(\mathrm{K}$ edge). In this case, the $6.40 \mathrm{keV}$ lines is generated via photoionization, and the continuum is due to Thomson scattering. The EW in the X-ray scenario depends on the angle $\theta$ between the line of sight and the incident photon direction [ [Q]. Figure $\square$ shows the EW as a function of photon index with the reflection angle $\theta=90^{\circ}$, which gives the maximum EW. The $6.40 \mathrm{keV}$ line produced by the X-ray irradiation has the EW below $1 \mathrm{keV}$ and can be distinguished from the proton origin.

\section{Result and Discussion}

\subsection{Eastern Region of Galactic Center}

We have performed the $6.40 \mathrm{keV}$ line survey on the Galactic plane $|l|<4^{\circ}$ near the Galactic center using the Suzaku satellite, and investigated the intensity distribution of the iron lines at 


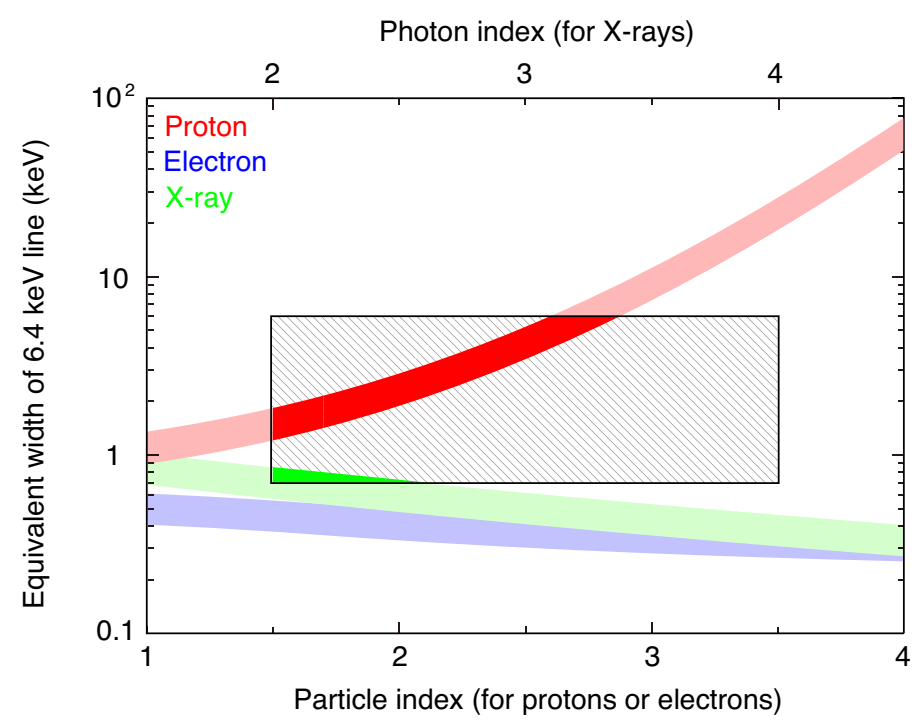

Figure 1: Equivalent widths of $6.40 \mathrm{keV}$ for the $\mathrm{X}$-ray, LECR electron, and LECR proton models shown with green, blue, and red lines, respectively [ [Q, Q $]$ ]. The solid rectangle indicates the constrained parameter space for the eastern region of Galactic center.

6.40, 6.68, and $6.97 \mathrm{keV}$, respectively. The detailed analysis is written in [ए0]. The 6.68 and $6.97 \mathrm{keV}$ lines have symmetrical distributions between the eastern (positive $l$ ) and western (negative l) sides within $|l|=1^{\circ} .5-3^{\circ} .5$. On the other hand, an asymmetrical distribution of the $6.40 \mathrm{keV}$ line intensity was found (figure $\square$ ). The symmetrical model with the dashed curves well represents the data in the western side, whereas the data points in the eastern side significantly exceed the same model. This inconsistency indicates that the excess $6.40 \mathrm{keV}$ line has a different origin from the $6.68,6.97 \mathrm{keV}$ lines. In order to examine the unknown origin, we compared the excess with the distribution of MCs. The red lines in figure $\square$ are the ${ }^{12} \mathrm{CO}$ line intensity taken by NANTEN, which nicely fills the residuals in the eastern side. The $6.40 \mathrm{keV}$ line should be emitted from the cold interstellar gases.

We, then, made an X-ray spectrum of the excess component and fit the spectrum with a power law plus a Gaussian line at $6.40 \mathrm{keV}$ (figure B). The best-fit photon index of the continuum and the EW are $3 \pm 1$ and $1.3 \pm 0.4 \mathrm{keV}$, respectively. If we take systematical uncertainty of statistical fluctuation of the symmetrical component into account, the EW value is constrained to be 0.7$5.9 \mathrm{keV}$. As is mentioned in Sec.2, the EW value is a key to distinguish the origin: X-rays, cosmicray protons, or electrons. The allowed parameter region is shown in figure $\mathrm{W}$. The electron origin is completely rejected. The X-ray origin is marginally accepted. Using the $6.40 \mathrm{keV}$ line flux and the total mass of the molecular gas in the area, we should require an X-ray irradiating source with a unrealistic luminosity $L_{X}=10^{40}(D / 450 \mathrm{pc})^{2} \mathrm{erg} \mathrm{s}^{-1}$, where $D$ is the distance of the $6.40 \mathrm{keV}$ line emitting region from the source. No source with such a luminosity is found in this area. Thus, the $\mathrm{X}$-ray origin would be unlikely.

In the case of the proton origin, adopting the spectral index of -2.5 from the best-fit result (see figureШ), we estimate the energy density of $\sim 80 \mathrm{eV} \mathrm{cm}^{-3}$ in $0.1-1000 \mathrm{MeV}$. This value is 


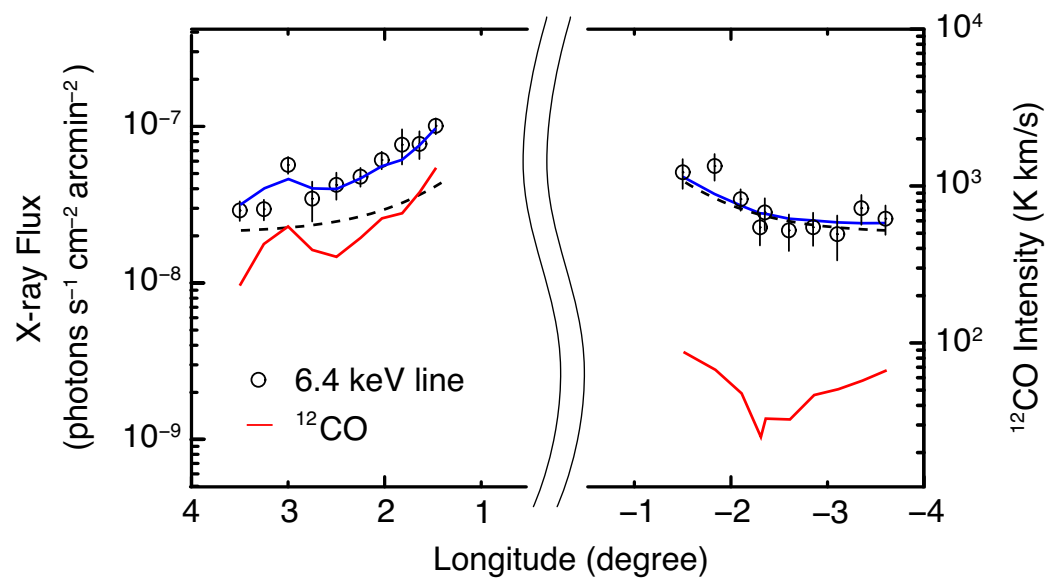

Figure 2: Intensity profile of the $6.40 \mathrm{keV}$ line (circle) overlaid with the ${ }^{12} \mathrm{CO}$ intensity (red) [U0]. The dashed lines well represents the distribution of the $6.40 \mathrm{keV}$ line in the western side. A symmetric model cannot explain the eastern side. The blue lines are the sum of the symmetric distribution model and the ${ }^{12} \mathrm{CO}$ intensity multiplied by a constant factor.

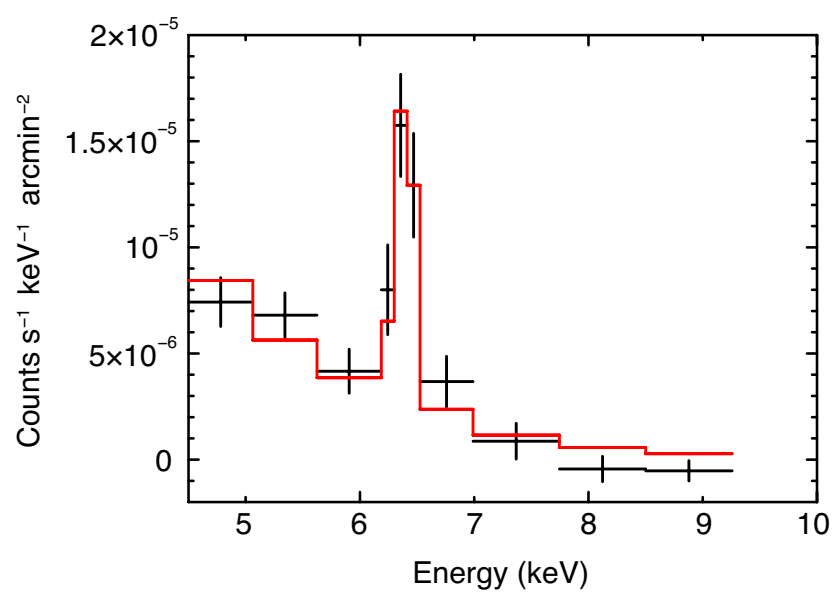

Figure 3: X-ray spectrum of the excess component in the eastern region of Galactic center. The solid line consists of a continuum with $\Gamma=3$ and the $6.40 \mathrm{keV}$ line with the equivalent width of $1.3 \mathrm{keV}$.

about two orders of magnitude higher than the average value $1 \mathrm{eV} \mathrm{cm}^{-3}$ of the cosmic rays [U]]. The diffusion length of $\mathrm{MeV}$ protons is very short, and thus the $\mathrm{MeV}$ protons should be produced in situ. There is no possible accelerating source, such as a SNR, a pulsar wind nebula, or a highlyactive black hole. Furthermore, since the excess $6.40 \mathrm{keV}$ emission distributes about 2 degree, corresponding to $300 \mathrm{pc}$ (at $8 \mathrm{kpc}$ ), acceleration by a single known object would be difficult. In the previous observations of the MCs, the gas velocity has a quite large dispersion of $\sim 100 \mathrm{~km} \mathrm{~s}^{-1}$ [ए2]. In such a case, Alfvénic turbulence may occur and results in stochastic acceleration [ए33].

\subsection{Galactic SNRs}

SNRs are the most plausible source accelerating the Galactic cosmic rays. Our measurement 
of LECRs with the $6.40 \mathrm{keV}$ line should be valid for SNRs. In fact, analyzing the Suzaku data of 3C391 and Kes79, [14, [15] reported detection of the $6.40 \mathrm{keV}$ line which would originated from LECRs. We expanded the search for the $6.40 \mathrm{keV}$ line to other SNRs located in the eastern region of the Galactic ridge, where 3C 391 and Kes 79 are located. We selected five SNRs (W28, W44, Kes 67, Kes 69, and Kes 78) which interact with MCs and carefully analyzed the archive Suzaku data in the $5-10 \mathrm{keV}$ band. Detailed results will be reported by Nobukawa et al. (in prep).

We made the $6.40 \mathrm{keV}$ line band image (figure 1 ). Non X-ray background was subtracted and the vignetting effect was corrected. One can see local enhancements of the $6.40 \mathrm{keV}$ line in all the five SNRs. The SNRs are located on the Galactic plane $\left(5^{\circ}<l<40^{\circ},|b|<1^{\circ}\right)$, and hence the hard X-ray band above $5 \mathrm{keV}$ is dominated by the Galactic background, i.e. the GRXE. We examined the spectra extracted from the regions where the $6.40 \mathrm{keV}$ line emission is enhanced and their surrounding regions by fitting with a model consisting of a power law and Gaussian lines and measured the intensities of the $6.40 \mathrm{keV}, 6.68 \mathrm{keV}$, and $6.97 \mathrm{keV}$ lines. Comparing the iron K-shell lines between the enhanced and the surrounding regions, we found no excess in other iron lines than the $6.40 \mathrm{keV}$ line. The significances of the $6.40 \mathrm{keV}$ line enhancement are $1.6 \sigma, 2.7 \sigma, 2.9 \sigma$, $2.0 \sigma$, and $2.0 \sigma$ for W28, Kes 67, Kes 69, Kes 78, and W44, respectively.

SNRs can have an iron line at $\sim 6.4 \mathrm{keV}$ originated from high temperature plasma in low ionization state $n t<10^{9} \mathrm{~s} \mathrm{~cm}^{-3}$. The enhance of the $6.40 \mathrm{keV}$ line detected in this study can originate from the rarely ionized iron ejecta. However, the SNRs concerned are in a dense environment and interact with dense MCs (typically $n>10^{3} \mathrm{~cm}^{-3}$ ), and hence the plasma easily reaches a high ionization state in their evolution [16]. Furthermore, whereas SNRs from which the iron K-shell line is detected have higher electron temperature of $>1 \mathrm{keV}$ [ए]], All our samples have SNR plasma with electron temperature of $<1.0 \mathrm{keV}$ [ए], [1, [9, [20] (Since no previous report is available for Kes 67, we analyzed the spectrum and obtained the temperature of $0.4 \pm 0.1 \mathrm{keV}$ ). On the other hand, since all the SNRs are associated with MCs, a natural explanation is the interaction between high-energy particles (X-rays or cosmic rays) and the MCs.

Because the spectrum extracted from each SNR had limited statistics, we co-added the spectra extracted from the enhanced and the surrounding regions, respectively, to study the origin of the $6.40 \mathrm{keV}$ line quantitatively. We, then, subtracted the latter spectrum from the former one. In the same manner as the eastern region of the Galactic center (Sec. 3.2), the spectrum was fitted with a model of a power law plus the $6.40 \mathrm{keV}$ line, resulting in the photon index of 1.5-8 and the equivalent width of $1.0_{-0.4}^{+0.7} \mathrm{keV}$. Assuming the iron abundance in the interstellar medium on the Galactic ridge of 1.0 solar, the electron origin cannot explain the result (see the lower limit of each line in figure $\mathbb{W}$ ). In the case of the X-ray origin, a source with the luminosity $L_{X} \sim$ $10^{38}(D / 100 \mathrm{pc})^{2} \mathrm{erg} \mathrm{s}^{-1}$ is required. No such a bright source is observed in the vicinity of the five SNRs. Therefore, the enhanced $6.40 \mathrm{keV}$ line would come from the LECR protons.

We investigate a correlation between the morphologies of the $6.40 \mathrm{keV}$ line emission and MCs (figure (1). In Kes 69 and W44, the $6.40 \mathrm{keV}$ line and the MCs are well correlated, while it is not the case in other three samples. Although the spatial resolution is not good, HI clouds were detected in W28 and Kes 67. Their distributions seem to coincide with the peaks of the $6.40 \mathrm{keV}$ line emission. For Kes 78, no good HI data have been taken. Using the averaged value of the column density $N_{\mathrm{H}}=10^{22} \mathrm{~cm}^{-2}$, the energy density of the LECR protons is calculated to be at least $10-100 \mathrm{eV} \mathrm{cm}^{-3}$. Due to the Lorentz force and ionization loss, $\mathrm{MeV}$ protons hardly diffuse. 

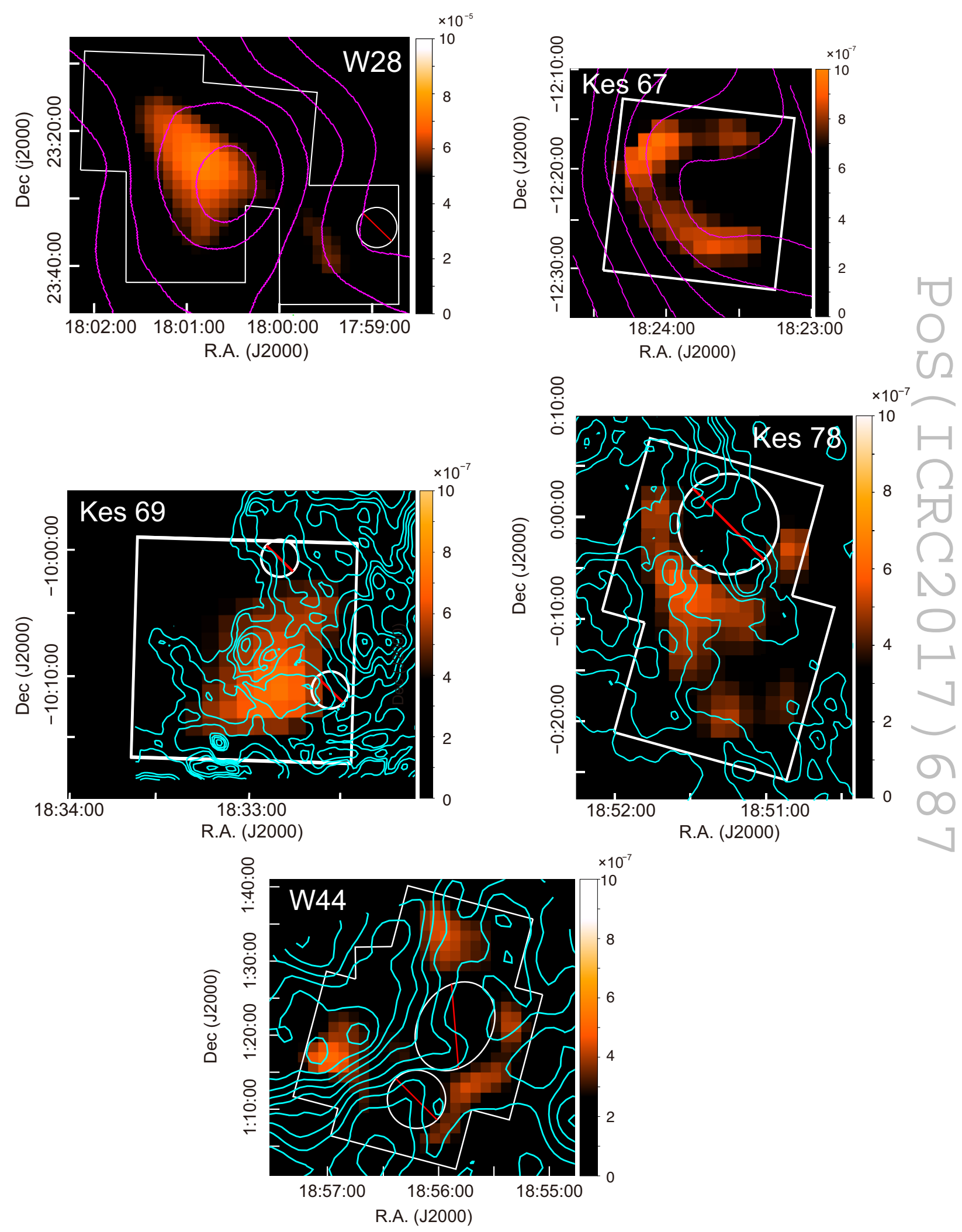

Figure 4: $6.40 \mathrm{keV}$ line emission map (color) of the five SNRs. The contours are $\mathrm{HI}$ at $37.5 \mathrm{~km} \mathrm{~s}^{-1}$, HI at $18.1 \mathrm{~km} \mathrm{~s}^{-1},{ }^{13} \mathrm{CO}(J=1-0)$ at $80-84 \mathrm{~km} \mathrm{~s}^{-1}$, and ${ }^{12} \mathrm{CO}(J=2-1)$ at $40.0-50.3 \mathrm{~km} \mathrm{~s}^{-1}$, for W28, Kes 67, Kes 69, Kes 78, and W44, respectively [21, 22, 231, 24, 25]. 
The $6.40 \mathrm{keV}$ line emission should trace the site where $\mathrm{MeV}$ protons are generated by a shock interacting with the MCs or HI clouds.

\section{Conclusion}

We discovered the $6.40 \mathrm{keV}$ line produced by LECR protons from the five SNRs (W28, Kes 67, Kes 69, Kes 78, W44, in addition to the previous detection in 3C391, Kes 79), and the eastern region of the Galactic center, with the energy densities of $>10-100 \mathrm{eV} \mathrm{cm}^{-3}$ and $\sim 80 \mathrm{eV} \mathrm{cm}^{-3}$, respectively. For the SNRs, the incident LECR protons are generated in situ, where the shock front contacts with MCs or HI clouds, and then produce the $6.40 \mathrm{keV}$ line. On the other hand, in the eastern region of the Galactic enter, there is neither SNR, a pulsar wind nebula, nor a highly active black hole. Stochastic acceleration by Alfvén turbulence is a possible origin for the LECR protons.

\section{Acknowledgement}

This research was supported by a grant from the Hayakawa Satio Fund awarded by the Astronomical Society of Japan. KKN is supported by Research Fellowships of the JSPS for Young Scientists.

\section{References}

[1] K. Koyama, R. Petre, E.V. Gotthelf, U. Hwang, M. Matsuura, M. Ozaki, \& S.S. Holt, Evidence for Shock Acceleration of High-Energy Electrons in the Supernova Remnant SN1006, Nature 378 (6554) 255-258.

[2] M. Ackermann et al., Detection of the Characteristic Pion-Decay Signature in Supernova Remnants, Science 339 (6) 807-811.

[3] E.C. Stone, A.C. Cummings, F.B. McDonald, B.C. Heikkila, N. Lal, \& W.R. Webber, Voyager 1 Observes Low-Energy Galactic Cosmic Rays in a Region Depleted of Heliospheric Ions, Science, 341 (6142) 150-153.

[4] D.M. Worrall, F.E. Marshall, E.A. Boldt, \& J.H. Swank, HEAO 1 measurements of the galactic ridge, ApJ, 255111.

[5] S. Yamauchi, et al., Iron Emission Lines on the Galactic Ridge Observed with Suzaku, PASJ, 61 (sp1) S225-S232.

[6] S. Yamauchi, K.K. Nobukawa, M. Nobukawa, H. Uchiyama, \& K. Koyama, Scale heights and equivalent widths of the iron K-shell lines in the Galactic diffuse X-ray emission, PASJ 68 (4) 59.

[7] M. Nobukawa, H. Uchiyama, K.K. Nobukawa, S. Yamauchi, \& K. Koyama, Origin of the Galactic Diffuse X-Ray Emission: Iron K-shell Line Diagnostics, ApJ 833 (2), 268.

[8] V. Dogiel, D. Chernyshov, K. Koyama, M. Nobukawa, \& K.-S. Cheng, K-Shell Emission of Neutral Iron Line from Sagittarius B2 Excited by Subrelativistic Protons, PASJ, 63 (3) 535-541.

[9] M. Tsujimoto, Y. Hyodo, \& K. Koyama, Suzaku spectroscopic study of hard X-ray emission in the Arches cluster, PASJ, 59 (sp1) S229-S235. 
[10] K. K. Nobukawa, M. Nobukawa, H. Uchiyama, T. G. Tsuru, K. Torii, T. Tanaka, D. O. Chernyshov, Y. Fukui, V. A. Dogiel, \& K. Koyama, Enhancement of the $6.4 \mathrm{keV}$ Line in the Inner Galactic Ridge: Proton-induced Fluorescence?, ApJL, 807 (1) article.id L10.

[11] A. Neronov, D. V. Semikoz, \& A. M. Taylor, Low-Energy Break in the Spectrum of Galactic Cosmic Rays, PhRvL, 108 (5) id 051105.

[12] K. Torii, et al., A Detailed Observational Study of Molecular Loops 1 and 2 in the Galactic Center, PASJ, 62 (5) 1307-1332.

[13] T. Amano, K. Torii, T. Hayakawa, \& Y. Fukui, Stochastic Acceleration of Cosmic Rays in the Central Molecular Zone of the Galaxy, PASJ, 63 (6) L63-L66.

[14] T. Sato, K. Koyama, T. Takahashi, H. Odaka, \& S. Nakashima, Discovery of recombining plasma in the supernova remnant 3C 391, PASJ, 66 (6) article.id 1249.

[15] T. Sato, K. Koyama, S-H. Lee, \& T. Takahashi, Suzaku spectra of a Type-II supernova remnant, Kes 79, PASJ, 68 (SP1) article.id S8.

[16] H. Yamaguchi, et al., Discriminating the Progenitor Type of Supernova Remnants with Iron K-shell Emission, ApJL, 785 (2) L27.

[17] Sawada, M., \& Koyama, K. X-Ray Observations of the Supernova Remnant W28 with Suzaku. I. Spectral Study of the Recombining Plasma, PASJ, 64 (4) 81.

[18] Bocchino, F., et al. A population of isolated hard X-ray sources near the supernova remnant Kes 69, $A \& A, 541$ A 152.

[19] Bamba, A., et al. Discovery of X-Ray Emission from the Galactic Supernova Remnant G32.8-0.1 with Suzaku, ApJ, 818 (1) 63.

[20] Uchida, H., et al. Recombining Plasma and Hard X-Ray Filament in the Mixed-Morphology Supernova Remnant W 44, PASJ, 64 (6) 141.

[21] Velázquez, P. F., Dubner, G. M., Goss, W. M., \& Green, A. J. Investigation of the Large-scale Neutral Hydrogen near the Supernova Remnant W28, ApJ, 124 (4) 2145-2151.

[22] Dubner, G., Giacani, E., Reynoso, E., Goss, W. M., Roth, M., \& Green, A. Interaction of the Supernova Remnant G18.8+0.3 with the Surrounding Medium, ApJ, 118 (2) 930-941.

[23] X. Zhou, Y. Chen, Y. Su, \& J. Yang, Discovery of Molecular Shells Associated with Supernova Remnants. I. Kesteven 69, ApJ, 691 (1) 516-524.

[24] P. Zhou, \& Y. Chen, Molecular Environment and an X-Ray Spectroscopy of Supernova Remnant Kesteven 78, ApJ, 743 (1) 4.

[25] S. Yoshiike et al., The Neutral Interstellar Gas toward SNR W44: Candidates for Target Protons in Hadronic $\gamma$-Ray Production in a Middle-aged Supernova Remnant, ApJ, 768 (2) 179. 